\title{
Algebraic correlations and anomalous fluctuations in ordered flocks of Janus particles fueled by an AC electric field
}

\author{
Junichiro Iwasawa $\odot,{ }^{1,2, *}$ Daiki Nishiguchi $\odot,,^{1, \dagger}$ and Masaki Sano $\odot^{2,3, *}$ \\ ${ }^{1}$ Department of Physics, The University of Tokyo, Hongo 7-3-1, Tokyo, 113-0033, Japan \\ ${ }^{2}$ Universal Biology Institute, Graduate School of Science, The University of Tokyo, Hongo 7-3-1, Tokyo, 113-0033, Japan \\ ${ }^{3}$ Institute for Natural Sciences, School of Physics and Astronomy, Shanghai Jiao Tong University, Shanghai 200240, China
}

(Received 30 August 2021; accepted 12 October 2021; published 10 November 2021)

\begin{abstract}
We study the polar collective dynamics of Janus colloidal particles fueled by an AC electric field. When the density is high enough, the polar interactions between the particles induce a polar orientationally ordered state, which exhibits features reminiscent of the Vicsek model such as true long-range order and giant number fluctuations. Independent measurements of the polarity and velocity at the single particle level allowed us to investigate the single particle dynamics within the ordered state. We discovered theoretically unaddressed statistical properties of the ordered state such as the asymmetric relation of polarity and velocity, enhanced rotational diffusion stronger than in the disordered state, and an algebraic autocorrelation of the polarity. Our experimental findings, at the crossroads of the Vicsek physics and the active Brownian particles physics, shed light on the so-far-unexplored physics arising from the interplay between the polarity and the velocity.
\end{abstract}

DOI: 10.1103/PhysRevResearch.3.043104

\section{INTRODUCTION}

Active matter systems consume and dissipate energy at the level of their local units to generate systematic motion based on their internal degrees of freedom (e.g., polarities) [1-4]. After decades of extensive research, it is now known that active matter systems can exhibit a wide range of phenomena such as phase separation [5-8], active chain formation $[9,10]$, active turbulence [11-14], etc. Among these topics, orientational order in active matter has gained considerable attention since the introduction of the Vicsek model in 1995, where pointwise polar particles move at constant speed toward their polarity and try to align their polarities with local neighbors under the presence of noise [15]. Theoretical work concerning the Vicsek-style models has been done including particle, kinetic, and hydrodynamic levels [16-24]. In particular, the phenomenological hydrodynamic equations by Toner, Tu, Ramawsamy, and their coworkers have predicted the existence of true long-range order and giant number fluctuations (GNF) for the orientationally ordered state $[16-18,23,25]$. In these theoretical and numerical studies, each particle's polarity is assumed to be parallel to its velocity. In experimental systems, on the other hand, particles do not necessarily move toward their polarities due to thermal and/or athermal noise and interparticle (hydrodynamic, excluded volume, or electrostatic)

\footnotetext{
*jiwasawa@ubi.s.u-tokyo.ac.jp

†nishiguchi@noneq.phys.s.u-tokyo.ac.jp

†sano.masaki@sjtu.edu.cn
}

Published by the American Physical Society under the terms of the Creative Commons Attribution 4.0 International license. Further distribution of this work must maintain attribution to the author(s) and the published article's title, journal citation, and DOI. interactions (e.g., systems of elongated bacteria [26], vibrated polar disks [27], motility assay [28], and active rolling colloids $[29,30])$.

One of the minimal models for investigating such active systems whose particle velocities do not necessarily coincide with their polarities is the active Brownian particles (ABP) model. In the ABP model, particles self-propel with a constant self-propulsive force under rotational/translational noise. The particles interact only by volume exclusion and do not exhibit any aligning interactions $[31,32]$. In spite of the absence of alignment, intriguing collective phenomena such as motilityinduced phase separation have been reported for ABP [33]. However, it still remains unclear what lies between nonaligning ABP-like systems and the aligning Vicsek-style models. Active colloidal systems similar to the ABP model have been experimentally realized and studied by using, e.g., autocatalytic colloidal particles in hydrogen peroxide and rolling colloids under a DC electric field. However, active particles fueled by chemicals do not exhibit ordered states reminiscent of those in Vicsek-style models so far [6,7,34]. In the case of rolling colloids, because the particles do not possess intrinsic polarity and their polarities are only electronic and invisible, in-depth study of the interplay between the polarity and velocity in the ordered state is not possible [30]. Thus, our understanding of how polarity and velocity interact with each other and how they behave in orientationally long-range ordered colloidal systems with hydrodynamic interactions remains elusive. For example, in the systems of elongated bacteria and motility assay, although true long-range order has been observed, particles could only be detected in a coarsegrained manner [26,28].

Janus particles [35], which are colloidal particles with two distinct hemispheres, offer a great platform for investigating active matter experimentally [6,8,9,32,36-39]. Among 
these Janus particles, the ones fueled by an AC electric field are especially suitable for analysis from the viewpoint of statistical physics because they constantly convert energy into motion without running out of energy sources and thus, allow long enough measurements for statistical analysis $[8,9,32,37,39,40]$, which is not the case for autocatalytic particles due to the consumption of chemical fuels. Under an AC electric field, Janus particles display two different mechanisms of swimming depending on the field frequency: induced charge electrophoresis (ICEP) at low frequency [41-43]; self-dielectrophoresis (sDEP) at high frequency [44,45]. Importantly, the two swimming mechanisms induce motion from the dielectric hemisphere to the metallic hemisphere $[8,9,32,37,38,40,43]$ or vice versa $[9,38,40,45]$, which allows us to define and observe each particle's polarity based on the location of its metallic hemisphere [8,9]. Not only the swimming mechanisms but also the interactions between particles depend on the field frequency and ion concentration, which lead to various types of collective behavior such as a turbulent phase [37], a swarming state with vortices [38], flagella like movement [9], and active clustering [8]. Thus, Janus particles provide us an ideal venue for experimentally studying the collective dynamics of ordered systems while keeping track of polarity at the single particle level.

Here in this paper, we perform experiments on the quasitwo-dimensional system of active Janus particles fueled by an $\mathrm{AC}$ electric field. We specifically use the AC field frequency regime where Janus particles swim by sDEP and exhibit a flocking phase. Through the extraction of both the polarity and velocity for each particle, we show that this flocking phase of Janus particles can exhibit true long-range orientational order. This ordered state of Janus particles also exhibited statistical features such as GNF and algebraic correlations for orientation fluctuations, which are reminiscent of the predictions of Toner and $\mathrm{Tu}[16,17,23]$. We further investigate the space-time correlations of density fluctuations leading to an estimate of dynamical and anisotropy exponents for the ordered state of Janus particles. In addition, through single particle measurements, we show that the particles counterintuitively exhibit larger diffusion in the ordered state than that in the disordered state for short time scales. We suggest that this anomalous enhanced diffusion stems from the coupling between particle polarity and velocity through computations of the cross-correlation function, which is a dismissed feature in Vicsek-style models. Our work, through the observation of both velocities and polarities, unravels hitherto unexplored statistical properties within orientationally ordered active colloidal systems.

\section{EXPERIMENTAL SYSTEM}

Our experimental system is composed of silica-based Janus particles with $3.17 \mu \mathrm{m}$ diameter [9]. To create distinct hemispheres, $35 \mathrm{~nm}$ of titanium followed by $15 \mathrm{~nm}$ of silica were deposited to a monolayer of silica colloidal particles (Bangs Laboratories, Inc., SS05N) using electron beam deposition and thermal evaporation, respectively. The additional $15 \mathrm{~nm}$ layer of silica was deposited to suppress oxidization and the subsequent changes of electrical properties and color (from black to transparent) of the Ti surface. After deposition, the

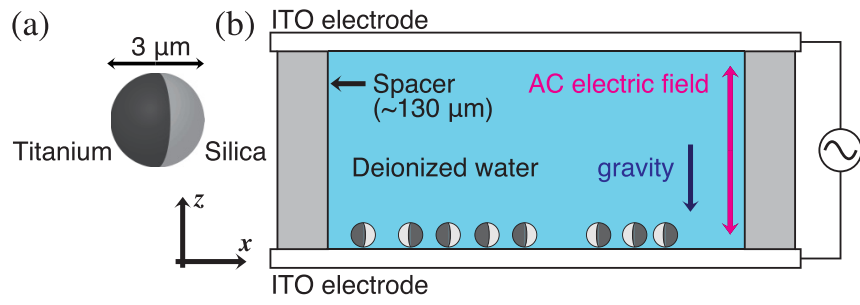

FIG. 1. Schematic image of the experimental system. (a) A silica-based Janus particle with a metallic titanium hemisphere. (b) The suspension of Janus particles were sandwiched by two ITO coated electrodes. The particles sediment to the surface of the ITO electrode, which leads to quasi-two-dimensional motion on the $x y$ plane.

particles were suspended into deionized water and washed by sonication to prevent particles from forming clusters. This washing procedure was repeated three times after waiting for 30-60 min for particle sedimentation. The washed Janus particles were suspended in deionized water and then sandwiched by two $25-\mathrm{nm}$ silica-deposited indium tin oxide (ITO) coated electrodes (Mitsuru Optical Co. Ltd.), separated by $130 \mu \mathrm{m}$ thick double-sided tape. Before applying the AC electric field, we waited for a few minutes to ensure that the particles have sedimented to the surface of the bottom electrode, which leads to a quasi-two-dimensional (quasi-2D) system of Janus particles (Fig. 1). Accordingly, the induced-charge electro-osmotic flow caused by the electric field lets the particle's polarity turn to the horizontal $x y$ plane, leading to confined motion in the horizontal 2D plane [47]. Note, because the particles have an induced dipole distribution of charges, which is parallel to the electric field, the particles will form clusters in the vertical direction as in Ref. [48] if the electric field was applied before the particles were completely sedimented to the bottom surface. It is also important to ensure that the Janus particles have both high enough density and large enough radius so that they do not escape from the quasi-two-dimensional plane due to vertical Brownian motion.

A sinusoidal AC electric field with $f=1 \mathrm{MHz}$ frequency and $16 \mathrm{~V}_{\mathrm{pp}}$ voltage was applied for all experiments, where $\mathrm{V}_{\mathrm{pp}}$ denotes the peak-to-peak voltage. In this frequency regime, it is known that Janus particles tend to move toward its metallic hemisphere, which contradicts with the ICEP theory [41-43]. This was first observed in Ref. [40], and now an explanation is given by the sDEP theory $[44,45]$. sDEP explains the metallic directed motion as a result of a surface force, arising from localized nonuniform electric field gradients between the asymmetric particle and the surface of the electrode. Unlike traditional dielectrophoresis where the motion is caused by the asymmetry of the external environment (e.g., electrode geometry), sDEP arises from the proximity of the asymmetric Janus particle itself to the conducting electrode surface [44,45]. Note, the ordinary electrophoretic effects caused by the negative charge of the silica particles could be negligible in our system since we use an AC electric field. Therefore, ordinary electrophoresis alone cannot compete with the ICEP/sDEP mechanism. Experiments were performed under two different density conditions: the low-density regime where 
(a)
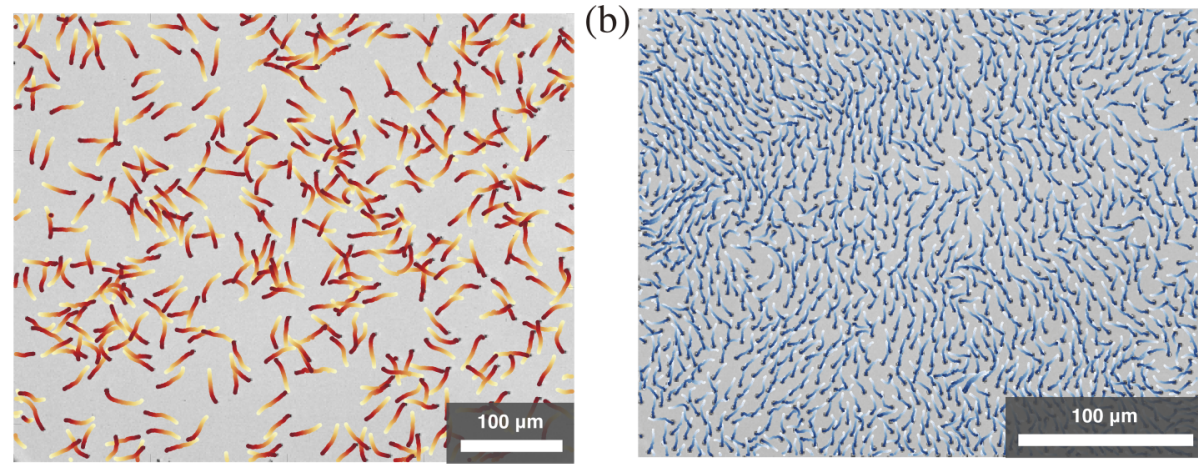

(c)

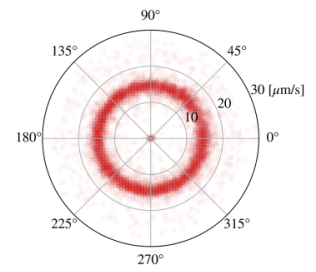

(d)

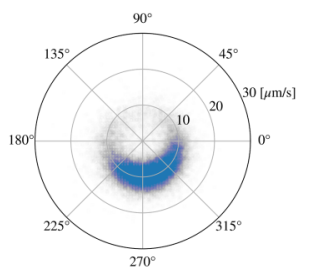

(e)

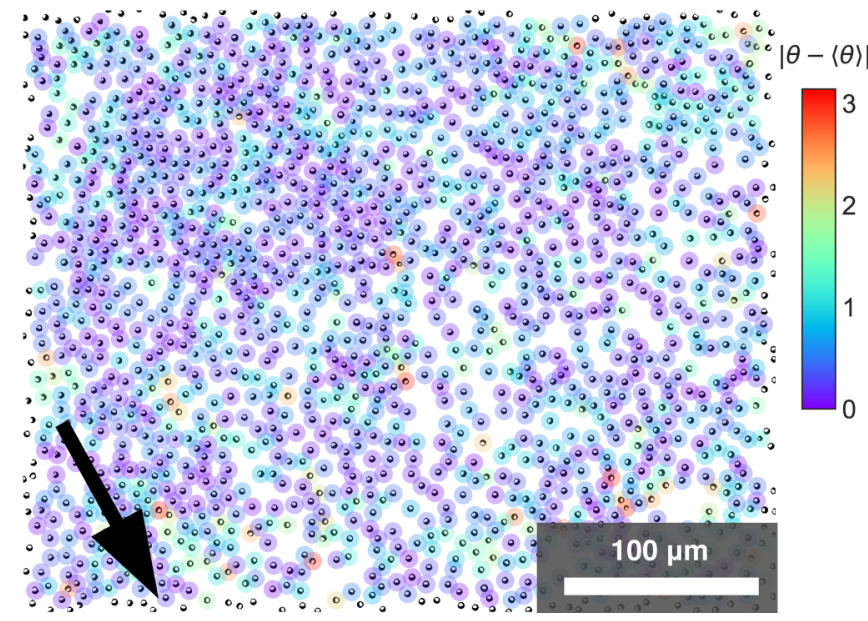

FIG. 2. Dynamics of Janus particles in the disordered and ordered states. (a), (b) Trajectories of the particles in the disordered state (3 s) and the ordered state $(2 \mathrm{~s})$, respectively. The bright and dark colors indicate the early and late times of each trajectory, respectively. (c), (d) Velocity distribution of the particles in the disordered (9909 particles) and ordered state (40205 particles), respectively [46]. The mean speeds for the disordered and ordered state were $\bar{v}_{d}=14.6 \mu \mathrm{m} / \mathrm{s}$ and $\bar{v}_{o}=8.5 \mu \mathrm{m} / \mathrm{s}$, respectively. (e) Binarized experimental snapshot of the Janus particles in the ordered state. Each particle is colored based on the deviation of its polarity from the global order $|\theta-\langle\theta\rangle|$ (radian). The black arrow shows the direction of global order $\langle\theta\rangle$.

$\rho \simeq 0.78$ particles $/ 1000 \mu \mathrm{m}^{2}$, and the high-density regime where $\rho \simeq 13$ particles $/ 1000 \mu \mathrm{m}^{2}$.

The observations of Janus particles were done using an inverted microscope (Nikon ECLIPSE TE2000-U) with objective lens (Nikon Plan Fluor ELWD, 20×, NA $=0.45$ and $40 \times, N A=0.60$ for the low- and high-density regime, respectively). A halogen lamp was used to illuminate the particles and a green filter was inserted in the light path before the sample to increase the contrast of the obtained images. Under this condition, the polarity of each Janus particle can be distinguished using its dark gray hemisphere indicating the titanium side, and the light gray hemisphere indicating the silica-only side (for example see movies 2, 3 in the Supplemental Material [46]). The dynamics were recorded at $10 \mathrm{fps}$ and $15 \mathrm{fps}$ for the low- and high-density regime, respectively, using a CMOS camera [Baumer LXG80, $3000 \times$ 2400 pixels $\simeq 430 \mu \mathrm{m} \times 340 \mu \mathrm{m}(40 \times)]$. This allowed us to capture the large-scale statistics of the particles while detecting the polarities and velocities of each particle at the same time.

Image analysis was performed using TRACKPY [49] and IMAGEJ [50]. Specifically, the polarities of the particles were detected by taking the difference of the centroid and center of mass of image intensity for each particle using the built-in algorithms in IMAGEJ [46]. The duration of the analyzed movies were $360 \mathrm{~s}$ and $212 \mathrm{~s}$ for the low- and high-density regime, respectively.

\section{RESULTS}

\section{A. Binary polar interaction and following motion}

To probe the interaction of the Janus particles in our system, we first measured the relative polarity distribution $P(\Delta \theta)$ for all Janus particle pairs in the low-density regime [Fig. 2(a)]. To examine the local interactions of the particles, particle pairs within $6 \mu \mathrm{m}$ and $30 \mu \mathrm{m}$ distance were extracted, respectively, and the relative polarity angle $\Delta \theta$ was measured for each pair [Figs. 3(a) and 3(b)] [46]. The results show that particles within $6 \mu \mathrm{m}$ range had a narrower distribution with a peak near $\Delta \theta=0$, which suggests the existence of polar interaction within the Janus particles. Indeed, when observing two Janus particles within a short distance, following motion where one particle tends to follow the other particle could often be observed [for example, see Fig. 3(c)].

The observed polar interaction and following motion can be understood as a result of the balance of electrostatic and hydrodynamic interactions between the particles. Although the interaction mechanism of Janus particles under an AC electric 

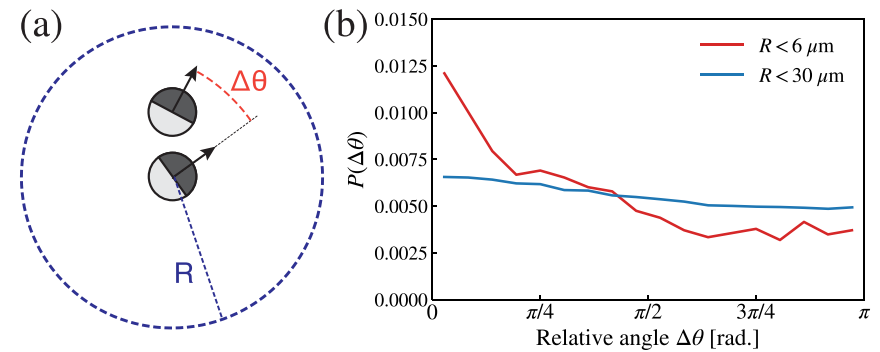

(c)

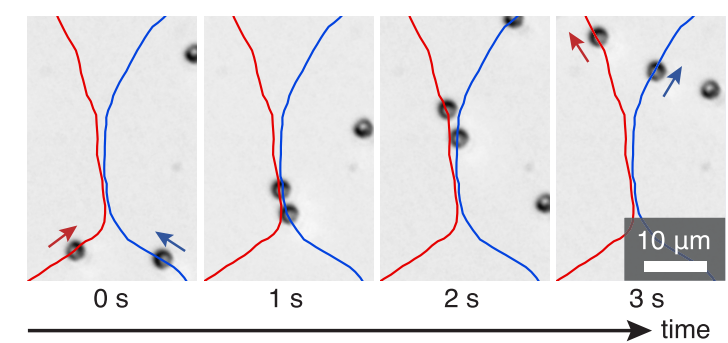

FIG. 3. Statistics of binary interactions of Janus particles. (a) Schematic picture describing the relative angle of particle pairs. (b) The relative polarity angle $\Delta \theta$ distribution of Janus particles in a low-density situation. $R$ denotes the distance of two isolated Janus particles. (c) Typical trajectories of two Janus particles when they are in close distance.

field is still under discussion $[9,38]$, our previous study experimentally validated the existence of polar interaction induced by the effective electrostatic dipole-dipole interaction between two particles both in the ICEP and the sDEP regimes [9]. Note that although our study in the sDEP regime uses a similar AC field frequency as in Ref. [9], the smaller ion concentration in this experiment than in our previous study generates a longer screening length (Debye length) $\lambda_{D} \propto 1 / \sqrt{c}$, where $c$ is the ion concentration [9]. The slip velocity $\boldsymbol{u}_{s}$ generated in the electric double layer scales as $\boldsymbol{u}_{s} \propto \lambda_{D}$, follows from the Helmholtz-Smoluchowski formula [41]. This results in a weak but relatively stronger pusher-type flow field than in our previous study [9], which prevents the particles from forming active chains in the current setup. It should also be noted that flow fields calculated based on pusher-type flow fields also show following motion due to the reorientation of the following particle, which is consistent with our observations [51,52]. This synergy of electrostatic and hydrodynamic interactions leads to polar alignment, allowing us to observe the ordered dynamics of Janus particles as we see below.

\section{B. True long-range order}

Janus particles exhibited distinct global patterns when we changed particle density. While Janus particles exhibited uncorrelated disordered motion in the low-density regime (Figs. 2(a) and 2(c) supplemental movie 1), orientationally ordered coherent motion was observed in the high-density regime [Figs. 2(b), 2(d), and 2(e), supplemental movies 3, 4] due to the local polar interaction. This density dependency of Janus particles is consistent with the previous experimental results of Ref. [38]. Note, if we compare the Janus particle system with Vicsek-style models, the density and the noise amplitude should be the basic factors, which control the transition from the disordered state to the ordered state. Although the effective noise amplitude, namely the Péclet number, could be adjusted by changing the particle's velocity via the AC electric field voltage, we have not been able to investigate this in the current study. This is because the particles easily adhere to the surface of the ITO electrode at higher voltages forbidding long time observation. Ideally, the effective noise amplitude could also be changed by using particles with different diameters, but it would be nearly impossible to continuously change the noise amplitude in this case. It should also be noted that the global orientation of the system varied each time we conducted the experiment, and thus, is not deterministic. This is analogous to the setting of the Vicsek-style models where the global orientation results from spontaneous symmetry breaking of the continuous rotational symmetry of the system.

To quantitatively assess the properties of orientational order in the low- and high-density regimes of the system, we computed the polar order parameter,

$$
C=\left\langle\left|\left\langle e^{i \theta}\right\rangle_{S}\right|\right\rangle_{t},
$$

for different sizes of rectangular (5:4) regions of interest (ROIs) taken in spatially uncorrelated locations (see Ref. [46] for details), where $\theta$ is the particle polarity and $\langle\cdot\rangle_{S}$ and $\langle\cdot\rangle_{t}$ denotes the average within the same ROI and statistically independent time frames, respectively (Fig. 4, [46]). In the low-density regime, the polar order parameter decays as a power law $C \propto 1 / \sqrt{S}$ [Fig. 4(a)]. This is consistent with the case with $N$ randomly oriented particles. In contrast, in the high-density regime, we can observe a decay slower than a power law within $S \sim 10^{5} \mu \mathrm{m}^{2}$. This asymptotic behavior characterizes the true long-range order, which was previously reported in Vicsek-style models [4] and in the bacterial experiment [26] where the orientational order parameter converges to a positive finite value. Note, the decay slower than a power law in our Janus particle system is observed for about 2.8 decades, which is larger than the range reported in the long-range ordered phase of bacteria [26] and even comparable to the range reported in numerical simulations (see Fig. 4(a) of Ref. [4]). To confirm the existence of true long-range order, we performed nonlinear fitting for the polar order parameter at $S<10^{5} \mu \mathrm{m}^{2}$, using the CURVE_ FIT function from the SCIPY package [53]. The data was in good fit with an algebraic convergence to a finite value $C-C_{\infty} \sim S^{-\gamma / 2}$ where $C_{\infty}=0.74$ and $\gamma / 2=0.53$ [see the inset of Fig. 4(b)], which indicates true long-range order in the system. It should be noted that recent simulations showed $\gamma=0.64$ for the Vicsek model [4]. At the same time, however, the decay of the order parameter deviates from this trend of algebraic convergence at $S>10^{5} \mu \mathrm{m}$. This is caused by the existence of counterflows of the particles (see supplemental movies 3,4 ), which is unavoidable due to the closed boundary conditions and the number conservation of the particles unless we elaborate an effective periodic boundary condition by, e.g., a racetracklike confinement [29]. However, in the case of our electrically driven Janus particles, microstructures fabricated by, e.g., photoresist distort the electric field, which leads to unwanted complex 3D electroconvection and destroys the two-dimensionality of the system. Therefore, we opted for using a simple system by sandwiching as large a droplet of 

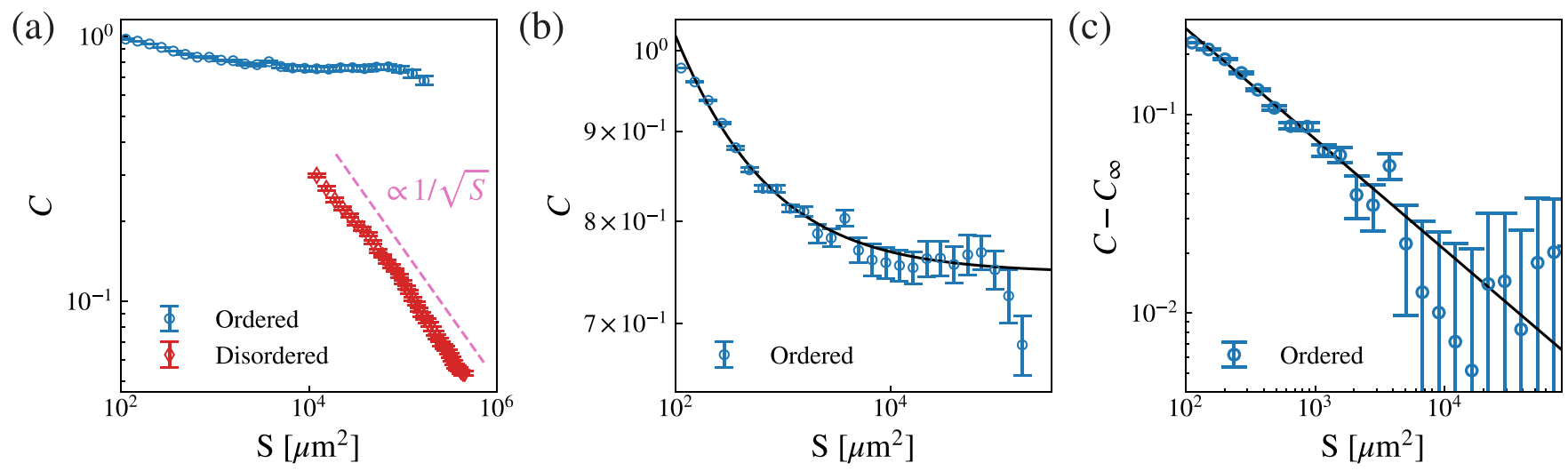

FIG. 4. The polar order parameter for the ordered and disordered states. (a) Log-log plot of the polar order parameter $C$ vs $S$, the area of the ROI. The blue and red points show the order parameter for the ordered and disordered state, respectively. (b) The same data as in (a) for the ordered state in a magnified range (log-log). (c) The same data as in (b) with the asymptotic value $C_{\infty}=0.74$ subtracted (log-log). The solid black line shows the nonlinear fit: $C=C_{\infty}+k S^{-\gamma / 2}$ with $C_{\infty}=0.74, k=3.1$, and $\gamma / 2=0.53$. The data for ROIs with $S>10^{5} \mu m^{2}$ have been excluded from the nonlinear fitting due to the existence of counterflows in the system. Error bars in (a), (b), (c): standard error.

the suspension of Janus particles as possible between the two electrodes. Overall, the finite size scaling analysis assures that the Janus particles exhibit polar collective motion with true long-range order.

\section{Interplay of polarity and velocity}

To investigate the detailed dynamics of single particle trajectories, we computed the polarity autocorrelation function $f_{p}(\tau)$ for the disordered and ordered states, respectively. Here,

$$
f_{p}(\tau)=\left\langle\boldsymbol{n}_{p}(t) \cdot \boldsymbol{n}_{p}(t+\tau)\right\rangle_{t},
$$

where $\boldsymbol{n}_{p}(t)$ denotes the polarity for the particle at time $t$, and $\langle\cdot\rangle_{t}$ takes the average for particle trajectories longer than $24 \mathrm{~s}$ [Figs. 5(a) and 5(b)]. We can first observe that the polarity autocorrelation decays exponentially in the disordered state. At small time scales, this decay was slightly faster than the theoretical estimation for an active Brownian particle $f_{p}(\tau)=$ $\exp \left(-2 D_{r} \tau\right)$ [31,36], where $1 / D_{r} \sim 24.2 \mathrm{~s}$ is the rotational diffusion constant [Fig. 5(a)] from the Einstein-Stokes relation, which is due to the polarity detection errors [32]. We thus focus on the time scale $\tau=0-12 \mathrm{~s}$, where the decay of the autocorrelation exhibited a slope of $21.15 \pm 0.08 \mathrm{~s}$, close to that of the theoretical estimation.

In the ordered state, on the other hand, $f_{p}(\tau)$ exhibited a slow power-law-like decay in the long time scale $(\tau>1 \mathrm{~s}$, [46]). Since particle polarities in the ordered state tend to align to the global order, we suspected that this power-law behavior of $f_{p}(\tau)$ is related to the fluctuations of the global order. To assess this hypothesis, we compared the connected polarity autocorrelation function in the ordered state with that of the global order (Fig. 6). In Fig. 5(c), we show the connected polarity (velocity) autocorrelations,

$$
\begin{aligned}
& f_{p, C}(\tau)=\left\langle\Delta \boldsymbol{n}_{p}(t) \cdot \Delta \boldsymbol{n}_{p}(t+\tau)\right\rangle_{t}, \\
& f_{v, C}(\tau)=\left\langle\Delta \boldsymbol{n}_{v}(t) \cdot \Delta \boldsymbol{n}_{v}(t+\tau)\right\rangle_{t},
\end{aligned}
$$

where $\Delta \boldsymbol{n}_{p, v}(t)=\boldsymbol{n}_{p, v}(t)-\left\langle\boldsymbol{n}_{p, v}(t)\right\rangle$, and $\left\langle\boldsymbol{n}_{p, v}(t)\right\rangle$ is the direction of global order defined by polarity or velocity, respectively. A power-law-like decay with a slope close to -0.7 for $f_{p, C}(\tau)$ could be observed for both the polarity and velocity autocorrelations. Interestingly, the connected
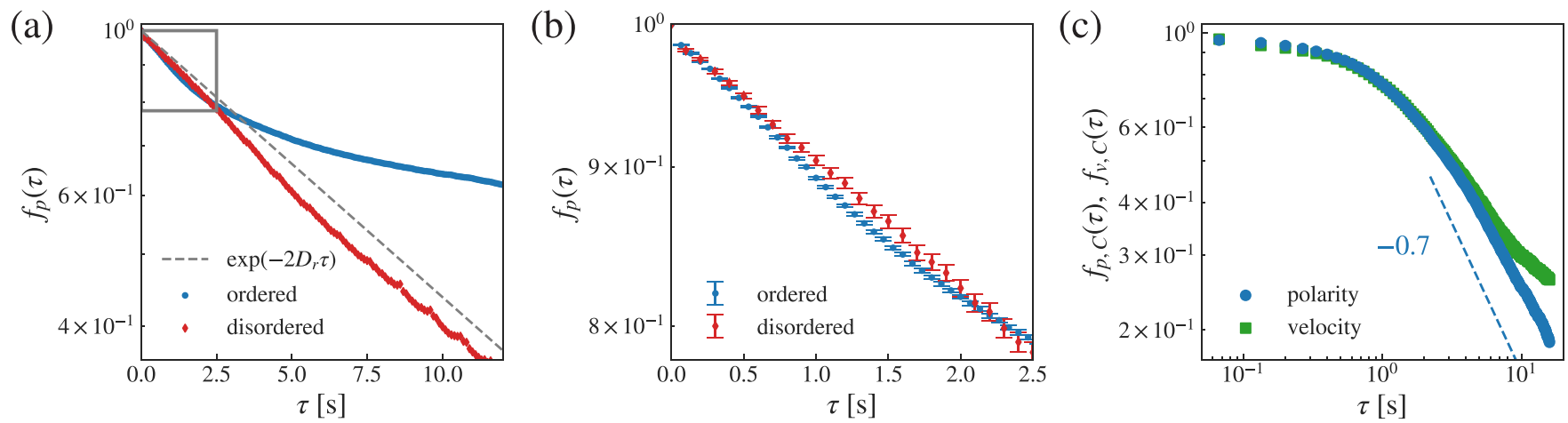

FIG. 5. Autocorrelation functions. (a) Polarity autocorrelation function in the ordered (red) and disordered state (blue) on a semilog scale. $\tau$ denotes the time delay. The dashed line shows $\exp \left(-2 D_{r} \tau\right)$ where $D_{r} \simeq 1 / 24.2 \mathrm{~s}^{-1}$ is the rotational diffusion constant for a $3.17 \mu \mathrm{m}$ diameter particle derived from the Einstein-Stokes relation. (b) The same data as in (a) in a magnified view, which is shown as a gray box in (a) (semilog). Error bars show the standard error. (c) The connected autocorrelation functions for polarity: $f_{p, C}(\tau)$ and velocity: $f_{v, C}(\tau)$ in the ordered state. The blue dashed line with a slope: -0.7 is a guide for the eye. 

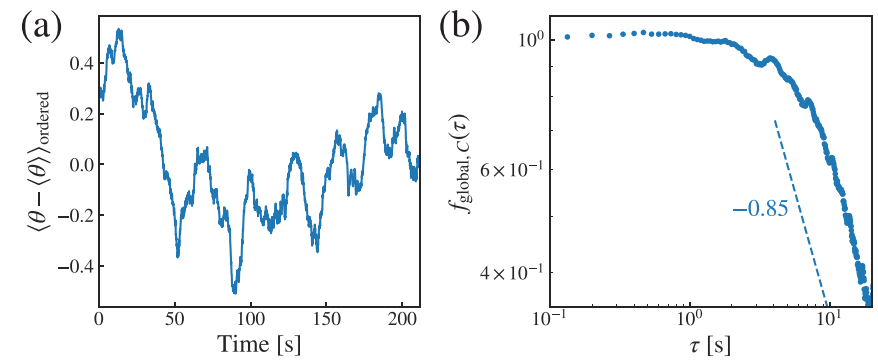

FIG. 6. Fluctuations of the mean polarity in the ordered region. (a) Time series of the fluctuations of global order $\langle\theta-\langle\theta\rangle\rangle_{\text {ordered }}$ where $\langle\cdot\rangle_{\text {ordered }}$ takes the average within the highly ordered region $\left(S<10^{5} \mu \mathrm{m}\right)$. (b) Autocorrelation of the polarity fluctuations from global order $f_{\text {global }, C}(\tau)=\left\langle\Delta \tilde{\boldsymbol{n}}_{p}(t) \cdot \Delta \tilde{\boldsymbol{n}}_{p}(t+\tau)\right\rangle$, where $\Delta \tilde{\boldsymbol{n}}_{p}(t)=$ $\left\langle\boldsymbol{n}_{p}-\left\langle\boldsymbol{n}_{p}\right\rangle_{t, \text { ordered }}\right\rangle_{\text {ordered }}$. The blue dashed line with a slope: -0.85 is a guide for the eye.

autocorrelation of the global order $f_{\text {global }, C}(\tau)$ [46] also showed a power-law-like decay with a slope of -0.85 , close to that of $f_{p, C}(\tau)$ [Fig. 6(b)]. Although there is, to the best of our knowledge, no theory predicting the slope for these power-law decays in global order and polarity (velocity) autocorrelations, these observations support our hypothesis that the power-law behavior of the polarity autocorrelations is a consequence of the fluctuations in global order. However, it should be noted that in a finite system, the direction of global order diffuses such that the autocorrelation function decays exponentially [24]. Therefore, we should expect that the power-law behavior of $f_{\text {global, } C}(\tau)$ that we observe is a transient to an exponential decay. Indeed, when we focus on longer time scales $(\tau>$ $10 \mathrm{~s}$ ), we can observe a decay faster than a power law for $f_{\text {global }, C}(\tau)$ [46]. Although the limited length scale of our experimental system hinders the long time behavior of single particle trajectories, we expect that the connected polarity autocorrelation function $f_{p, C}(\tau)$ will also show a crossover to an exponential decay, reflecting the fluctuations of the global order.

The polarity autocorrelation function for particles in the ordered state also exhibited peculiar behavior at short time scales $(\tau \sim 1 \mathrm{~s})$. Figure 5(b) shows a magnified view of the behavior of $f_{p}(\tau)$, and it can be observed that $f_{p}(\tau)$ of the ordered state decays faster than that of the disordered state at short time scales. Because this time scale is similar to the time scale of the mean-free time (distance to the nearest neighbor $\sim 7 \mu \mathrm{m}) /\left(\right.$ average velocity $\left.\bar{v}_{o}\right) \sim 0.8 \mathrm{~s}$, we speculated that this enhanced diffusion was caused by the frequent and continuous interactions among closely distanced particles in the ordered state. To confirm this speculation, we computed the polarity-velocity cross-correlation function,

$$
g_{p v}(\tau)=\left\langle\boldsymbol{n}_{p}(t) \cdot \boldsymbol{n}_{v}(t+\tau)\right\rangle_{t},
$$

where $\boldsymbol{n}_{v}(t)=\boldsymbol{v}(t) /|\boldsymbol{v}(t)|$ is the unit velocity vector at time $t$ (Fig. 7). The assessment of $g_{p v}(\tau)$ revealed the asymmetric relation of polarity and normalized velocity in the ordered state, where polarity tended to correlate the most with the velocity of $0.2 \mathrm{~s}$ in the past $(\tau \sim-0.2)$. In other words, the polarity in the ordered state tended to follow the direction of the velocity of the particle with a finite time delay. It should be noted that, although not statistically significant, $g_{p v}(\tau)$ in the disordered
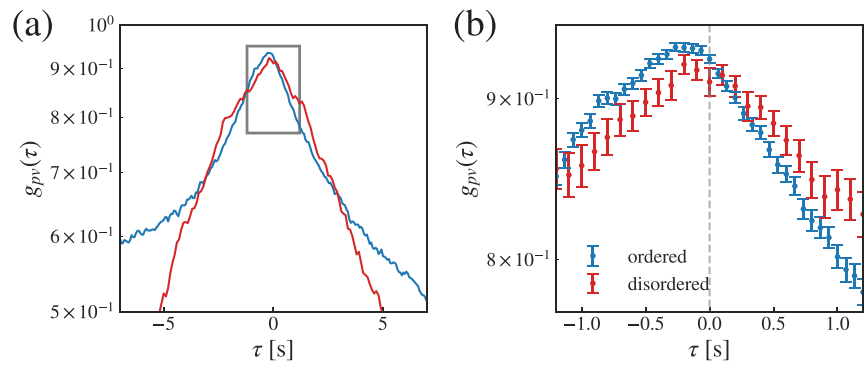

FIG. 7. Polarity-velocity cross-correlation functions. (a) The cross-correlation function $g_{p v}(\tau)$ for Janus particles in the ordered (blue) and disordered state (red). (b) The same data as in (a) in a magnified view for the gray box shown in (a). Error bars: standard error. The difference in error bar size is caused by the sparsity in the disordered state.

state showed a weak, if any, asymmetry around $\tau=0$ as well. These results imply that the asymmetry in $g_{p v}(\tau)$ is amplified in higher-density situations. We therefore suspect that this asymmetry is caused by electrostatic and/or hydrodynamic torques induced by collisions between individual particles. Overall, our results suggest that the particle polarities tend to follow the particle's velocity, yet local interactions between nearby particles disturb their alignment causing a time delay especially at the high-density state. These effects might explain the enhanced diffusion observed in the ordered state.

\section{Giant number fluctuations in the ordered state}

Giant number fluctuations (GNF), where the variance of the number of particles $\Delta N^{2}$ grows faster than its mean $\langle N\rangle$ $\left(\Delta N^{2} \propto\langle N\rangle^{2 \alpha}\right.$ with $\left.2 \alpha>1\right)$ in long-range ordered phases, are a hallmark of the Toner-Tu phase $[16,17,23]$. Since its theoretical prediction by Toner, Tu, Ramaswamy, and their coworkers [16,17,25], the presence of GNF has been extensively studied in numerical systems [18,21,24,54]. However, in addition to experimental difficulty in realizing collective motion with true long-range order, experimental detection of GNF requires delicate treatment due to many possible pitfalls such as difficulty in disentangling fluctuations arising from, e.g., phase coexistence or boundary effects as critically discussed in Ref. [26]. Therefore, GNF as the hallmark of the Toner-Tu-Ramaswamy phase had remained undetected until recent experiments on a long-range ordered nematic phase of elongated bacteria [26] and then on the polar ordered phase of colloidal rollers [30]. Here in our Janus particles system, as we have confirmed the true long-range order, it is natural to question whether the ordered state shows anomalous number fluctuations.

To quantify number fluctuations, the mean $\langle N\rangle$ and standard deviations $\Delta N$ of the number of particles for different sizes of ROIs were calculated for statistically independent frames (Fig. 8). For the disordered state, normal number fluctuations with $\Delta N \propto\langle N\rangle^{0.5}$ were observed, which is consistent with the central limit theorem. In contrast, anomalous number fluctuations were observed in the ordered state where $\Delta N \propto\langle N\rangle^{\alpha}$ with $\alpha>0.5$. In fact, the estimated exponent was $\alpha=0.790(5)$ with $95 \%$ confidence level, which is very close to the theoretical prediction $\alpha=0.80[16,17]$ and the recent 


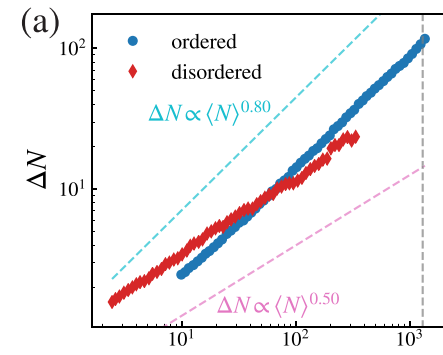

$\langle N\rangle$

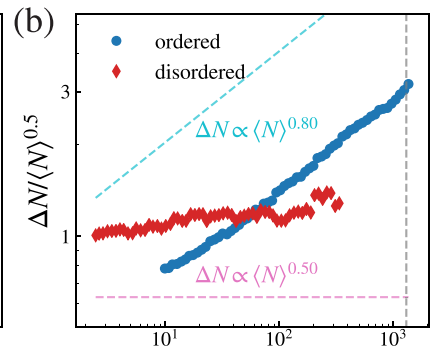

$\langle N\rangle$
FIG. 8. Scaling of number fluctuations. (a) Number fluctuations in the ordered (blue) and disordered (red) state. (b) Same data as in (a) with the number fluctuations normalized by $\langle N\rangle^{0.5}$. The cyan and magenta dashed lines show $\Delta N \propto\langle N\rangle^{0.8}$ and $\Delta N \propto\langle N\rangle^{0.5}$, respectively, as a guide to the eye. The gray dashed line indicates the scale of counterflows up to which the true long-range order persists.

numerical estimate $\alpha=0.84$ for the Vicsek model [24]. It is worth noting that the existence of nontrivial GNF has to be discussed under the presence of true long-range orientational order. Indeed, there are several experimental studies that report GNF for systems that are not in the fully ordered state (for details see the Supplemental Material of Ref. [26]). Here, we have set all ROIs to be placed at the center of the whole image so that the ROIs correspond to those that were used for the computation of the order parameter. Accordingly, the gray dashed line in Fig. 8 indicates the length scale $S \sim 10^{5} \mu \mathrm{m}^{2}$ at which the order parameter in the ordered phase starts to deviate from algebraic convergence to a finite value (i.e., true long-range order). Therefore, the observation of anomalous fluctuations below this line imply that ordered Janus particles do exhibit GNF in the context of the Toner-Tu phase.

\section{E. Algebraic scars of long-range order}

When a continuous symmetry is spontaneously broken, the entire ordered phase should also be characterized by an algebraic decay of its connected correlation functions [55]. In the case of the Vicsek model, a power-law decay for the orientation fluctuation correlation function exemplifies the spontaneous symmetry breaking of the rotational symmetry of the system, and its exponents have been predicted by the hydrodynamic theory of Toner and Tu [16,17,23]. Interestingly, a recent numerical study of the Vicsek model suggested that the ordered state could exhibit GNF exponents close to the conjectured values even though there existed a discrepancy in the exponents of the correlation functions [24]. Thus, in addition to number fluctuations, the fluctuation correlation functions are important measures to probe the statistical properties of orientational order in the system.

To address the questions of whether the orientational fluctuations of Janus particles exhibit scale-free correlations, and if so, whether they follow exponents of the Toner-Tu conjecture, we measured the orientation fluctuation correlation function,

$$
C_{C}(\boldsymbol{R}) \equiv\left\langle\left\langle\delta n_{p \perp}(t, \boldsymbol{r}) \delta n_{p \perp}(t, \boldsymbol{r}+\boldsymbol{R})\right\rangle_{\boldsymbol{r}}\right\rangle_{t},
$$

where $\delta n_{p \perp}(t, \boldsymbol{r})=\boldsymbol{n}_{\boldsymbol{p}}(t, \boldsymbol{r})-\left\langle\boldsymbol{n}_{\boldsymbol{p}}\right\rangle$ is the deviation of a single particle polarity $\boldsymbol{n}_{\boldsymbol{p}}$ from the global order $\left\langle\boldsymbol{n}_{\boldsymbol{p}}\right\rangle$, and $\langle\cdot\rangle_{\boldsymbol{r}}$ takes
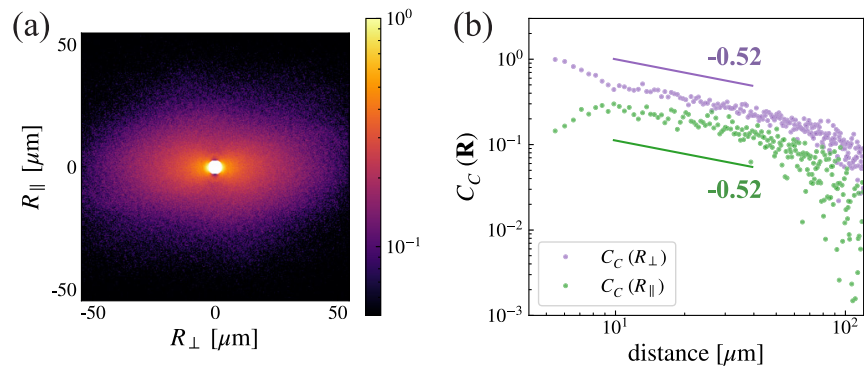

FIG. 9. Orientation fluctuation correlation function in the ordered state. (a) Color map of the correlation function $C_{C}(\boldsymbol{R})$. The global mean director is set aligned with the vertical axis $\left(R_{\|}\right)$. The region of $|\boldsymbol{R}|<6 \mu \mathrm{m}$ were excluded from calculations due to the small statistics. (b) $\log -\log$ plot of $C_{C}(\boldsymbol{R})$ both in the transverse $\left(R_{\perp}\right.$, purple) and longitudinal $\left(R_{\|}\right.$, green) direction. The purple and green solid lines are a guide for the eye, which show a slope of -0.52 . Note, the exponents from the Toner-Tu conjecture predict an anisotropic decay of $C_{C}(\boldsymbol{R}) \propto R_{\perp}^{-2 / 5}, R_{\|}^{-2 / 3}[16,17,23]$ and recent numerical simulations suggested $C_{C}(\boldsymbol{R}) \propto R_{\perp}^{-0.62}, R_{\|}^{-0.65}$ [24].

an average for all $|\boldsymbol{R}|$ distanced particle pairs (see Fig. 9). Here, the global order $\left\langle\boldsymbol{n}_{\boldsymbol{p}}\right\rangle$ was calculated by averaging the polarity $\boldsymbol{n}_{\boldsymbol{p}}$ both spatially and temporally. Figure 9(b) shows the decay of $C_{C}(\boldsymbol{R})$ in the transverse and longitudinal directions to global order. Although there is an anisotropy in the absolute value of the correlation function, it decays algebraically in both directions with the same exponent close to -0.52 . This algebraic decay signifies the spontaneous symmetry breaking of the rotational symmetry of the system and consequent scale-free features. However, the exponents of the decay differ from the Toner-Tu conjecture, which predicts an anisotropic decay of $C_{C}(\boldsymbol{R}) \propto R_{\perp}^{2 \chi}, R_{\|}^{2 \chi / \xi}$, where $\chi=-0.2$ and $\xi=0.6$ are the roughness and anisotropy exponent, respectively $[16,17,23]$. This discrepancy might stem from the difference in microscopic properties including steric and hydrodynamic interactions as we will discuss in the following section. Interestingly, recent experimental [30] and numerical [24] studies for polar orientational order have both reported weak, possibly vanishing, anisotropy for the orientational fluctuation correlation function. In addition, our analysis on the space-time correlations of density fluctuations also suggests weakly anisotropic sound modes propagating in our Janus colloidal flocks [46]. This leads to the estimate of the dynamical exponent $z \sim 1.2$ while the anisotropy exponent $\xi \sim 1$, suggesting a nondiffusive but weakly anisotropic dependence that qualitatively agrees with recent numerical simulations [24]. It should also be noted that the roughness exponent $\chi$ also takes similar but distinct exponents for theory, simulation, and experiments where $\chi=-0.20$ for the Toner-Tu conjecture [16,17,23], $\chi \sim-0.31$ for numerical simulations [24], $\chi \sim-0.38$ for active Quincke rollers [30], and $\chi \sim-0.26$ for our system of Janus particles. These differences in the exponents might indicate that some of the nonlinearities that were neglected in the original calculations [16,17] are relevant asymptotically, as suggested in Ref. [24]. Our measurements of $C_{C}(\boldsymbol{R})$ and the sound modes, along with recent numerical [24] and experimental [30] studies, may pave the way for further theoretical work on collective systems. 


\section{DISCUSSION}

We studied the collective motion of active Janus particles fueled by an AC electric field. At high density, the Janus particles exhibited polar orientational order, which allowed us to investigate and compare the statistical properties of the system with that of the Toner-Tu phase. We have confirmed GNF within the polar orientational ordered state with an exponent similar to but different from the theoretical predictions of Toner and Tu. In addition, an algebraic decay was observed for the orientation fluctuation correlation function, which is also associated with the spontaneous symmetry breaking of the rotational symmetry of the system. The observed exponents showed a discrepancy with the theoretical predictions and recent numerical simulations [24] of the Vicsek model, suggesting a different mechanism for the emergence of long-range order. As pointed out in Ref. [26] and then both experimentally and numerically verified in Refs. [28,56], weak volume exclusion that allows crossing over other particles is considered a key to make collective motion of elongated objects similar to the Vicsek-style interactions and thus achieve long-range order at least for the otherwise turbulent or clustering $2 \mathrm{D}$ nematic systems such as bacteria and gliding microtubules [26,28]. In the case of polar collective motion, the weak exclusion may not be a requirement for the long-range order because the particles cannot overlap each other in our experimental setup as in granular experiments [27,57]. What realizes long-range order in polar systems could be the following motion exemplified in Fig. 3, as discussed previously in, e.g., mutant Dictyostelium cells [58], and/or the torque aligning the polarity to the velocity, as studied in vibrated polar disks [59].

Our results might suggest that, although GNF could be observed, algebraic decay as in the Toner-Tu phases are difficult to observe in experimental systems as well as in numerical simulations. The exponents of the algebraic decay for the orientation fluctuation correlation function differed from that observed for polar flocks of Quincke colloidal rollers [30]. Although Janus particles and Quincke rollers are both considered as polar particles with polar interaction, the polarity acquisition mechanisms are completely different. Whereas the polarity of Janus particles is a priori defined by its metallic hemisphere, Quincke rollers acquire polarity by the spontaneous symmetry breaking of charge distribution on the particle scale. It could be interesting to investigate whether this difference in the polarity acquisition mechanism alters the global properties of the orientationally ordered system because the latter might have additional soft modes on the particle level.

Tracking both the velocity and polarity of all particles enabled the detailed assessment of the single particle dynamics both in the disordered and ordered states. Strikingly, the polarity autocorrelation showed a slow algebraic decay in the polar ordered state, which has never been studied even numerically. It could be interesting if this slow decay in polarity could be observed not only in active colloidal systems but also in collective biological matter [58].

Our results also suggest the interplay between the polarity and velocity of the particles, which might be explained by the hydrodynamic and/or the electrostatic torques between the particles. We have shown that this interplay is amplified in the high-density ordered state. This interplay between polarity and velocity is something that cannot be observed in the polar Vicsek model where the two vectors are identical and degenerated. A recent numerical study shows that the global properties could differ drastically by decoupling the polarity and velocity of the system [60]. Our polarity-resolved realization of the collective phase of self-propelled Janus particles can contribute to bridging the understandings on the two major active matter classes: the Vicsek physics and the active Brownian physics. Overall, our system of Janus particles provides a large-scale statistical study for polar ordered active colloidal particles, which opens a new avenue on further theoretical and experimental works for the field of active matter.

\section{ACKNOWLEDGMENTS}

We thank Benoît Mahault and Hugues Chaté for insightful discussions and valuable comments on an early version of the manuscript. We also thank Tetsuya Hiraiwa and Kazumasa A. Takeuchi for fruitful discussions. This work was supported by KAKENHI (Grant No. JP25103004). J.I. was supported by a Grant-in-Aid for the Japan Society for Promotion of Science Fellows (Grant No. JP18J21942). D.N. was supported by JSPS KAKENHI Grants No. JP19K23422, No. JP19H05800, and No. JP20K14426.
[1] S. Ramaswamy, The mechanics and statistics of active matter, Annu. Rev. Condens. Matter Phys. 1, 323 (2010).

[2] M. C. Marchetti, J. F. Joanny, S. Ramaswamy, T. B. Liverpool, J. Prost, M. Rao, and R. A. Simha, Hydrodynamics of soft active matter, Rev. Mod. Phys. 85, 1143 (2013).

[3] S. Ramaswamy, Active matter, J. Stat. Mech.: Theory Exp. (2017) 054002.

[4] H. Chaté, Dry aligning dilute active matter, Annu. Rev. Condens. Matter Phys. 11, 189 (2020).

[5] J. Tailleur and M. E. Cates, Statistical Mechanics of Interacting Run-and-Tumble Bacteria, Phys. Rev. Lett. 100, 218103 (2008).

[6] I. Theurkauff, C. Cottin-Bizonne, J. Palacci, C. Ybert, and L. Bocquet, Dynamic Clustering in Active Colloidal Suspensions with Chemical Signaling, Phys. Rev. Lett. 108, 268303 (2012).
[7] J. Palacci, S. Sacanna, A. P. Steinberg, D. J. Pine, and P. M. Chaikin, Living crystals of light-activated colloidal surfers, Science 339, 936 (2013).

[8] M. N. van der Linden, L. C. Alexander, D. G. A. L. Aarts, and O. Dauchot, Interrupted Motility Induced Phase Separation in Aligning Active Colloids, Phys. Rev. Lett. 123, 098001 (2019).

[9] D. Nishiguchi, J. Iwasawa, H.-R. Jiang, and M. Sano, Flagellar dynamics of chains of active Janus particles fueled by an AC electric field, New J. Phys. 20, 015002 (2018).

[10] J. Harder and A. Cacciuto, Hierarchical collective motion of a mixture of active dipolar janus particles and passive charged colloids in two dimensions, Phys. Rev. E 97, 022603 (2018). 
[11] H. P. Zhang, A. Be'er, E. L. Florin, and H. L. Swinney, Collective motion and density fluctuations in bacterial colonies, Proc. Natl. Acad. Sci. 107, 13626 (2010).

[12] K.-T. Wu, J. B. Hishamunda, D. T. Chen, S. J. DeCamp, Y.-W. Chang, A. Fernández-Nieves, S. Fraden, and Z. Dogic, Transition from turbulent to coherent flows in confined three-dimensional active fluids, Science 355, eaal1979 (2017).

[13] D. Nishiguchi, I. S. Aranson, A. Snezhko, and A. Sokolov, Engineering bacterial vortex lattice via direct laser lithography, Nature Commun. 9, 4486 (2018) .

[14] H. Reinken, D. Nishiguchi, S. Heidenreich, A. Sokolov, M. Bär, S. H. L. Klapp, and I. S. Aranson, Organizing bacterial vortex lattices by periodic obstacle arrays, Commun. Phys. 3, 76 (2020).

[15] T. Vicsek, A. Czirók, E. Ben-Jacob, I. Cohen, and O. Shochet, Novel Type of Phase Transition in a System of Self-Driven Particles, Phys. Rev. Lett. 75, 1226 (1995).

[16] J. Toner and Y. Tu, Long-Range Order in a Two-Dimensional Dynamical XY Model: How Birds Fly Together, Phys. Rev. Lett. 75, 4326 (1995).

[17] J. Toner and Y. Tu, Flocks, herds, and schools: A quantitative theory of flocking, Phys. Rev. E 58, 4828 (1998).

[18] S. Ramaswamy, R. Aditi Simha, and J. Toner, Active nematics on a substrate: Giant number fluctuations and long-time tails, Europhys. Lett. 62, 196 (2003).

[19] G. Grégoire and H. Chaté, Onset of Collective and Cohesive Motion, Phys. Rev. Lett. 92, 025702 (2004).

[20] E. Bertin, M. Droz, and G. Grégoire, Boltzmann and hydrodynamic description for self-propelled particles, Phys. Rev. E 74, 022101 (2006).

[21] H. Chaté, F. Ginelli, G. Grégoire, and F. Raynaud, Collective motion of self-propelled particles interacting without cohesion, Phys. Rev. E 77, 046113 (2008).

[22] E. Bertin, M. Droz, and G. Grégoire, Hydrodynamic equations for self-propelled particles: Microscopic derivation and stability analysis, J. Phys. A: Math. Theor. 42, 445001 (2009).

[23] J. Toner, Reanalysis of the hydrodynamic theory of fluid, polarordered flocks, Phys. Rev. E 86, 031918 (2012).

[24] B. Mahault, F. Ginelli, and H. Chaté, Quantitative Assessment of the Toner and Tu Theory of Polar Flocks, Phys. Rev. Lett. 123, 218001 (2019)

[25] J. Toner, Y. Tu, and S. Ramaswamy, Hydrodynamics and phases of flocks, Ann. Phys. (NY) 318, 170 (2005).

[26] D. Nishiguchi, K. H. Nagai, H. Chaté, and M. Sano, Long-range nematic order and anomalous fluctuations in suspensions of swimming filamentous bacteria, Phys. Rev. E 95, 020601(R) (2017).

[27] C. A. Weber, T. Hanke, J. Deseigne, S. Léonard, O. Dauchot, E. Frey, and H. Chaté, Long-Range Ordering of Vibrated Polar Disks, Phys. Rev. Lett. 110, 208001 (2013).

[28] S. Tanida, K. Furuta, K. Nishikawa, T. Hiraiwa, H. Kojima, K. Oiwa, and M. Sano, Gliding filament system giving both global orientational order and clusters in collective motion, Phys. Rev. E 101, 032607 (2020).

[29] A. Bricard, J.-B. Caussin, N. Desreumaux, O. Dauchot, and D. Bartolo, Emergence of macroscopic directed motion in populations of motile colloids, Nature (London) 503, 95 (2013).

[30] D. Geyer, A. Morin, and D. Bartolo, Sounds and hydrodynamics of polar active fluids, Nature Mater. 17, 789 (2018).
[31] A. Callegari and G. Volpe, Numerical simulations of active brownian particles, in Flowing Matter, edited by F. Toschi and M. Sega (Springer International Publishing, Cham, 2019), pp. 211-238.

[32] A. Poncet, O. Bénichou, V. Démery, and D. Nishiguchi, Pair correlation of dilute active brownian particles: From lowactivity dipolar correction to high-activity algebraic depletion wings, Phys. Rev. E 103, 012605 (2021).

[33] M. E. Cates and J. Tailleur, Motility-induced phase separation, Annu. Rev. Condens. Matter Phys. 6, 219 (2015).

[34] F. Ginot, I. Theurkauff, F. Detcheverry, C. Ybert, and C. CottinBizonne, Aggregation-fragmentation and individual dynamics of active clusters, Nature Commun. 9, 696 (2018).

[35] P. G. de Gennes, Soft matter, Rev. Mod. Phys. 64, 645 (1992).

[36] H.-R. Jiang, N. Yoshinaga, and M. Sano, Active Motion of a Janus Particle by Self-Thermophoresis in a Defocused Laser Beam, Phys. Rev. Lett. 105, 268302 (2010).

[37] D. Nishiguchi and M. Sano, Mesoscopic turbulence and local order in janus particles self-propelling under an ac electric field, Phys. Rev. E 92, 052309 (2015).

[38] J. Yan, M. Han, J. Zhang, C. Xu, E. Luijten, and S. Granick, Reconfiguring active particles by electrostatic imbalance, Nature Mater. 15, 1095 (2016).

[39] T. Mano, J.-B. Delfau, J. Iwasawa, and M. Sano, Optimal runand-tumble-based transportation of a Janus particle with active steering, Proc. Natl. Acad. Sci. 114, E2580 (2017).

[40] R. Suzuki, H. R. Jiang, and M. Sano, Validity of Fluctuation Theorem on Self-Propelling Particles, arXiv:1104.5607.

[41] T. M. Squires and M. Z. Bazant, Induced-charge electroosmosis, J. Fluid Mech. 509, 217 (2004).

[42] T. M. Squires and M. Z. Bazant, Breaking symmetries in induced-charge electro-osmosis and electrophoresis, J. Fluid Mech. 560, 65 (2006).

[43] S. Gangwal, O. J. Cayre, M. Z. Bazant, and O. D. Velev, Induced-Charge Electrophoresis of Metallodielectric Particles, Phys. Rev. Lett. 100, 058302 (2008).

[44] A. M. Boymelgreen and T. Miloh, Induced-charge electrophoresis of uncharged dielectric spherical janus particles, Electrophoresis 33, 870 (2012).

[45] A. Boymelgreen, G. Yossifon, and T. Miloh, Propulsion of active colloids by self-induced field gradients, Langmuir $\mathbf{3 2}$ 9540 (2016).

[46] See Supplemental Material at http://link.aps.org/supplemental/ 10.1103/PhysRevResearch.3.043104 for details for the experimental methods.

[47] M. S. Kilic and M. Z. Bazant, Induced-charge electrophoresis near a wall, Electrophoresis 32, 614 (2011).

[48] S. Gangwal, O. J. Cayre, and O. D. Velev, Dielectrophoretic assembly of metallodielectric janus particles in ac electric fields, Langmuir 24, 13312 (2008)

[49] D. B. Allan, T. Caswell, N. C. Keim, and C. M. van der Wel, TRACKPY: Trackpy v0.4.1, 2018.

[50] C. A. Schneider, W. S. Rasband, and K. W. Eliceiri, Nih image to imagej: 25 years of image analysis, Nature Methods 9, 671 (2012).

[51] E. Lauga and T. R. Powers, The hydrodynamics of swimming microorganisms, Rep. Prog. Phys. 72, 096601 (2009).

[52] J. S. Guasto, R. Rusconi, and R. Stocker, Fluid mechanics of planktonic microorganisms, Annu. Rev. Fluid Mech. 44, 373 (2012). 
[53] P. Virtanen, R. Gommers, T. E. Oliphant, M. Haberland, T. Reddy, D. Cournapeau, E. Burovski, P. Peterson, W. Weckesser, J. Bright et al., Scipy 1.0: Fundamental algorithms for scientific computing in python, Nature Methods 17, 261 (2020).

[54] F. Ginelli, F. Peruani, M. Bär, and H. Chaté, Large-Scale Collective Properties of Self-Propelled Rods, Phys. Rev. Lett. 104, 184502 (2010).

[55] F. Ginelli, The physics of the vicsek model, Eur. Phys. J.: Spec. Top. 225, 2099 (2016).

[56] X.-q. Shi and H. Chaté, Self-Propelled Rods: Linking Alignment-Dominated and Repulsion-Dominated Active Matter, arXiv:1807.00294.

[57] H. Soni, N. Kumar, J. Nambisan, R. K. Gupta, A. Sood, and S. Ramaswamy, Phases and excitations of active rod-bead mixtures: Simulations and experiments, Soft Matter 16, 7210 (2020).

[58] M. Hayakawa, T. Hiraiwa, Y. Wada, H. Kuwayama, and T. Shibata, Polar pattern formation induced by contact following locomotion in a multicellular system, eLife 9, e53609 (2020).

[59] K.-D. N. T. Lam, M. Schindler, and O. Dauchot, Self-propelled hard disks: Implicit alignment and transition to collective motion, New J. Phys. 17, 113056 (2015).

[60] B. Mahault, X.-c. Jiang, E. Bertin, Y.-q. Ma, A. Patelli, X.-q. Shi, and H. Chaté, Self-Propelled Particles with Velocity Reversals and Ferromagnetic Alignment: Active Matter Class with Second-Order Transition to Quasi-Long-Range Polar Order, Phys. Rev. Lett. 120, 258002 (2018). 